\title{
Clinicopathologic Study of Endometrial and Hormonal Changes after Metformin Therapy in Patients with Polycystic Ovary Syndrome \\ Salah-el-din Sayed Semary ${ }^{1}$, Al-Sayed A. Abd-Elrahman ${ }^{2}$, Ahmad Taha ${ }^{3}$, Mahmoud F.Midan ${ }^{4}$, Rashed M. Rashed ${ }^{4}$, Tarek M. Emran ${ }^{5}$, Tahra N ${ }^{4}$. \\ 1. Department of Pathology, Faculty of Medicine, Al-Azhar University, Damietta. \\ 2. Department of Anatomy and Embryology, Faculty of Medicine, Port Said University, Egypt. \\ 3. Department of Physiology, Faculty of Medicine, Port Said University, Egypt. \\ 4. Department of Obstetrics and Gynecology, Al-Azhar Faculty of Medicine, Damietta \\ 5. Department of Clinical Pathology, Al-Azhar Faculty of Medicine, Damietta \\ *Corresponding Author: Salah-el-din Sayed Semary , E-mail: drsalahsemary@yahoo.com
}

\begin{abstract}
Background: Polycystic ovary syndrome (PCOS) is a common complex genetic condition of women in the reproductive age. PCOS is a heterogeneous syndrome characterized by clinical/biochemical androgen excess, ovulatory dysfunction and polycystic ovaries. Metformin therapy has been proved to improve fertility in patients with PCOS, inducing not only high ovulation and pregnancy rates, but also reducing the incidence of miscarriages. Aim of the Work: This study aimed to evaluate the effects of metformin therapy on hormonal profile and endometrial tissue, including pattern of immunohistochemical expression of androgen receptors (AR), in patients with PCOS. Patients and Methods: 100 patients with PCOS were included in this study. Each investigated case was submitted to detailed medical history, clinical examination that included body hair distribution, body weight, height and body mass index (BMI), transvaginal ultrasound, laboratory investigations ( included fasting insulin, free testosterone, LH and FSH levels). Endometrial pipelle samples were taken for histopathological evaluation and assessment of androgen receptor expression. These investigations were done before and after three months of metformin treatment. Results: A significant decrease of BMI of the investigated cases after metformin therapy was observed ( $\mathrm{P}$ value $<0.003$ ). There was a significant decrease of LH level after metformin therapy from $9.17 \pm 2.84 \mathrm{Miu} / \mathrm{ml}$ to $6.18 \pm 3.6 \mathrm{Miu} / \mathrm{ml}$ and of fasting insulin level from $14.3 \pm 4.3$ to $8.2 \pm 5$.9. Insignificant increase of FSH level from $3.87 \pm 1.8$ to $4.85 \pm 2.6$ and also insignificant decrease of free testosterone level from $1.58 \pm 0.83$ to $1.38 \pm 0.44$ were also observed. Histopathological results of the endometrial specimens before metformin therapy revealed histologic features of early proliferative endometrium in 64 cases, 20 cases with a late proliferative endometrium and examination of the remaining 16 cases revealed features of simple endometrial hyperplasia. Among the 64 cases diagnosed as early proliferative endometrium before the therapy, 60 cases showed features of a late proliferative endometrium after treatment and the remaining 4 cases showed no histomorphologic changes. Among the 20 cases diagnosed as a late proliferative endometrium before therapy, 6 cases showed features of early secretory phase after therapy, 6 cases showed features of mid-secretory endometrium while the remaining 8 cases showed a late secretory endometrium that indicate successful ovulation after therapy. Regression of endometrial hyperplasia after therapy was noted in 6 of the 16 cases diagnosed as simple endometrial hyperplasia. Immunohistochemical (IHC) results revealed marked increase in endometrial AR expression in patients with PCOS compared to the normal fertile controls $(\mathrm{p}<0.004)$. Also, a significant decrease of AR expression in endometrial epithelial and stromal cells after metformin administration in patients with PCOS was noted $(p<0.003)$. Conclusion: Metformin therapy restores normal menstrual cyclicity in patients with PCOS, also, induces ovulation and showing significant decrease in endometrial AR expression.
\end{abstract}

Keywords: PCOS, Metformin, AR immunoexpression.

\section{INTRODUCTION}

Polycystic ovary syndrome (PCOS) is a common disorder of women in the reproductive age that characterized by clinical/biochemical manifestations and its main symptoms may appear from adolescence ${ }^{(1)}$. Various diagnostic criteria have been proposed, generally centered on the features of hyperandrogenemia, oligo-ovulation and polycystic ovarian morphology ${ }^{(\mathbf{1})}$. Insulin resistance is present in a majority of cases, with compensatory hyperinsulinemia contributing to hyperandrogenism via stimulation of ovarian androgen secretion and inhibition of hepatic sex hormone-binding globulin production ${ }^{(2)}$. Patients with PCOS usually need medical treatment for management of menstrualrelated disorders and infertility and also have various long-term complications which have been frequently underestimated ${ }^{(3)}$. Gestational diabetes and hypertensive disorders are more likely to occur in women with PCOS during pregnancy, and as they get older, metabolic diseases such as glucose intolerance, type II diabetes and hyperlipidemia are frequently occurred ${ }^{(3)}$. Therefore, lifelong follow-up 
and management for these patients are needed for detection and prevention of complications as early as possible ${ }^{(4)}$. Androgen receptors (AR) have been identified in the human endometrium but their role in endometrial cyclic development and function remain poorly understood (5). Although the proliferation and differentiation of endometrium are mainly mediated by oestrogen and progesterone receptors (ER \& PR), the AR may play some roles in modulating these changes, suggesting that $A R$ may be involved in physiological and pathological changes of the endometrium ${ }^{(6)}$. AR expression is primarily under androgenic control and high levels adversely affect priming and development of the endometrium in the follicular and luteal phases respectively ${ }^{(6)}$. Female androgen production depends on the adrenal glands and also on the expression of key enzymes in the endometrium that facilitate local androgen biosynthesis and conversion (6). Moreover, levels of circulating androgens, in women of reproductive age, fluctuate in a cycledependent manner and a mid-cycle peak is associated with conception ${ }^{(6)}$. AR and androgen signaling have a decisive role in the differentiation of human endometrial stromal cells into decidual cells ${ }^{(6)}$. Compelling evidence for androgen signaling in the regulation of endometrial function pertaining to implantation and pregnancy is provided by epidemiological studies that demonstrating a strong association between polycystic ovary syndrome, premature ovarian failure or advanced maternal age and adverse pregnancy outcome ${ }^{(7)}$. Thus, androgen signaling is an essential component of normal endometrial physiology and its perturbation is associated with reproductive failure ${ }^{(8)}$. In PCOS, ovarian hyperandrogenism, hyperinsulinemia from insulin resistance and altered intraovarian paracrine signaling can disrupt follicle growth ${ }^{(9)}$. The consequent follicular arrest in PCOS is accompanied by menstrual irregularity, anovulatory subfertility and accumulation of small antral follicles within the periphery of the ovary, giving it a polycystic morphology ${ }^{\left({ }^{10}\right)}$. Follicular arrest in PCOS develops when granulosa cells in antral follicles normally begin to express aromatase (at a size of $7 \mathrm{~mm}$ ), but excess intraovarian 5 $\alpha$-reduced androgens inhibit granulosa cell aromatase activity and impair follicle growth ${ }^{(9)}$. The frequent occurrence of associated hyperinsulinemia in PCOS further exacerbates ovarian follicular arrest, which promotes ovarian hyperandrogenism by stimulation of $17 \alpha-$ hydroxylase activity in theca cells (10). Hyperinsulinemia also amplifies luteinizing hormone-stimulated and insulin-like growth factor 1 (IGF-1)-stimulated androgen production, elevates serum testosterone levels through decreased hepatic sex hormone-binding globulin production, and enhances serum IGF-1 bioactivity through suppressed IGF-binding protein production (10). Insulin excess also promotes premature follicle luteinization through enhanced follicle-stimulating hormone-induced granulosa cell differentiation, which arrests granulosa cell proliferation and subsequent follicle growth (11). Metformin is considered an insulin-sensitizing drug and has a promise in therapy for patients with PCOS ${ }^{(12)}$. Metformin treatment administered in high doses $(3000 \mathrm{mg} /$ day) for a 6 months period was proved to be useful for weight loss, improvement of ovarian function and decrease of androgen levels in obese patients with PCOS ${ }^{(13)}$. Also, metformin therapy to PCOS patients decreases serum fasting insulin, total and free testosterone as well as estradiol levels at oocyte retrieval, enhances pregnancy and live birth rates, and also diminish the risk of severe ovarian hyperstimulation syndrome ${ }^{(\mathbf{1 3})}$. Metformin therapy may cause rare side effects such as nausea, which usually disappear after long-term therapy ${ }^{(\mathbf{1 4})}$.

\section{AIM OF THE WORK}

This study aimed to evaluate the effects of metformin therapy on hormonal profile and endometrial tissue in patients with PCOS, including pattern of immunohistochemical expression of androgen receptors (AR).

\section{PATIENTS AND METHODS}

This observational study was conducted on outpatient clinic of Obstetrics Gynecology and Pathology Departments of Al-Azhar University Hospital at New Damietta during the period from April 2016 to January 2018. An informed consent was taken from each woman participating in this study. The study was approved by the Ethics Board of Al-Azhar University. This study included 100 patients fulfilling the inclusion criteria: Inclusion criteria: 1. Patients at childbearing period (20-40 years). 2. Patients diagnosed as PCOS by Rotterdam criteria ${ }^{(15)}$, if 2 of the 3 features were present (oligo-ovulation or anovulation, hyperandrogenism \& PCO by U/S that showing 12 or more 2-9 mm ovarian follicles in one or two ovaries). 3. Use of metformin alone for 
3 consecutive months $(850 \mathrm{mg} /$ day $)$ and past history of infertility. Exclusion criteria: 1. Age $<20$ or $>40$ years. 2. Other causes of hyperandrogenemia. 3. Use of oral contraceptive pills or clomiphene citrate.

All the patients in the current study were subjected to menstrual history, drug history and any symptoms that are suggestive of PCOS including infertility, hirsutism and obesity. General examination including hair distribution all over the body was done according to the modified Ferriman Gallwey score ${ }^{(\mathbf{1 6})}$. This scoring system evaluates nine different body parts (upper lip, chin, chest, upper back, lower back, upper abdomen, lower abdomen, arm, and thigh). A score greater than 8 indicates hirsutism. Estimation of the body weight and height were assessed while the patient was standing and wear light clothes with no shoes. BMI was calculated as weight in $\mathrm{kg} / \mathrm{height}$ in $\mathrm{m}^{2}(<20$ considered underweight, 20-25; optimal weight, $>25$; overweight, >30; obese, > 35; moderate obesity, >40: morbid obesity). Transvaginal ultrsonography and laboratory investigations (including LH, FSH, fasting insulin level and free testosterone level) were done. Endometrial sampling by pipple aspirator for histopathological evaluation and assessment of AR expression by IHC were also done. These investigations were done before and after 3 months of metformin administration. Blood Sampling: $3 \mathrm{ml}$ of venous blood were collected from each patient at $9 \mathrm{AM}$ in a sterile vacationer tube, left for clotting and then centrifuged at 3000 r.p.m., the serum was separated and stored at $20^{\circ} \mathrm{C}$ till the time of hormonal assay. Hormonal assay principles: All assayed hormones (FSH, LH, fasting insulin and free testosterone levels) were done using an automated chemiluminscence immunoassay by immulite instruments (Diagnostic products corporation, Los Angeles, CA 90045 USA). Histopathological and IHC methods: Endometrial pipelle samples were received in Pathology Department, fixed in 10\% buffered formaldehyde solution, embedded in paraffin blocks, cut into 4 micron thick sections and stained with hematoxylin and eosin (H\&E). For immunohistochemical assay, tissue sections were taken on poly-L-lysine coated adhesive slides (Sigma, USA), incubated in $60^{\circ} \mathrm{c}$ for 30 minutes and then the sections were deparaffinized in xylene and rehydrated by alcohol. Antigen retrieval was achieved by heating slides at $95^{\circ} \mathrm{C}$ for $10-20$ minutes in $0.1 \mathrm{~mol} / \mathrm{L}$ citrate buffer $(\mathrm{pH} 6)$ using water bath. To block the endogenous peroxidase activity, sections were immersed in 3\% hydrogen peroxide for 30 minutes. Sections were incubated with anti-AR polyclonal antibody (dilution 1:500, Santa Cruz Biotechnology, CA) overnight at $4^{\circ} \mathrm{C}$. After applying secondary antibody (Biotinylated anti-rabbit immunoglobulin) associated with the Envision system, sections were washed in PBS and 3.3'-Diaminobenzidine tetrahydrochloride was used as chromogen and Mayer's hematoxylin as counterstaining. Immunoreactivity for AR was restricted to nuclei of the endometrial epithelial and stromal cells and appeared as a brownish coloration. By omitting the primary antibody, sections were used as the negative control. Prostatic tissue was used as external positive control. 5 endometrial specimens were added to the study from fertile patients presenting by fibroid uterus with unremarkable endometrial pathologic findings for comparison of androgen receptor (AR) expression (age ranged from 30 to 37 years). Early proliferative phase was defined as (days 4-7), mid proliferative (days 8 - 10) and late proliferative (days 11 -14) without signs of secretory changes. Histologic dating of secretory phase biopsies was assigned according to the criteria of Noyes ${ }^{(17)}$. The immunohistochemical evaluation was performed with the semiquantitative H-SCORE (Histo" score) which is applicable to androgen receptors. The final score was obtained by the following formula: $3 \times$ percentage of strongly stained nuclei $+2 \times \%$ of moderately stained nuclei $+\%$ of weakly stained nuclei. The final score of each case ranged from 0 to $300{ }^{(\mathbf{1 8})}$. Statistical analysis: The collected data were organized, tabulated and statistically analyzed using statistical package for social sciences (SPSS) V22. For quantitative data, mean standard deviation (SD), minimum and maximum were calculated. For comparison between two measurements, the independent samples $(t)$ test was used. Pearson correlation co-efficient (r-test) was used for correlating different variables. For all tests $\mathrm{p}$ value $<0.05$ were considered significant, while $\mathrm{p}$ value $>0.05$ were considered insignificant.

\section{RESULTS}

The mean age of the studied group was $29.1 \pm 5.7$ years, mean BMI was $25.3 \pm 0.27 \mathrm{~kg} / \mathrm{m}^{2}$ and parity ranged from 0 to 2 (Table 1). A significant decrease of BMI of the investigated 
cases after metformin therapy was observed (Table 2). A significant decrease of LH level from $9.17 \pm$ $2.84 \mathrm{Miu} / \mathrm{ml}$ to $6.18 \pm 3.6 \mathrm{Miu} / \mathrm{ml}$ and also a significant decrease in fasting insulin level from $14.3 \pm 4.3$ to $8.2 \pm 5.9$ after metformin therapy were also observed (Table 3). There was a significant decrease of $\mathrm{LH} / \mathrm{FSH}$ ratio in the studied cases after metformin administration as compared to before therapy (Table 4).

Table 1: Demographic data of studied cases

\begin{tabular}{|l|c|}
\hline \multicolumn{1}{|c|}{ Variable } & Mean \pm SD \\
\hline Age $($ years $)$ & $29.1 \pm 5.7$ \\
\hline BMI $\left(\mathrm{kg} / \mathrm{m}^{2}\right)$ & $25.3 \pm 0.27$ \\
\hline Parity & Range $(0-2)$ \\
\hline
\end{tabular}

Table 2: Comparison between the studied cases before and after metformin administration as regard BMI

\begin{tabular}{|l|c|c|c|}
\hline $\begin{array}{c}\text { Groups } \\
\text { Parameter }\end{array}$ & $\begin{array}{c}\text { Before } \\
\text { Treatment }\end{array}$ & $\begin{array}{c}\text { After } \\
\text { Treatment }\end{array}$ & $\begin{array}{c}\text { P- } \\
\text { value }\end{array}$ \\
\hline $\begin{array}{l}\mathrm{BMI}\left(\mathrm{kg} / \mathrm{m}^{2}\right) \\
\text { Mean } \pm \mathrm{SD}\end{array}$ & $25.3 \pm 0.27$ & $16.3 \pm 0.28$ & $<\mathbf{0 . 0 0 3}$ \\
\hline
\end{tabular}

$\mathrm{P}$ value $<0.003$ indicates significance

Table 3: Comparison between the studied cases before and after metformin administration as regard endocrine parameters

\begin{tabular}{|l|c|c|c|}
\hline \multicolumn{1}{|c|}{$\begin{array}{c}\text { Groups } \\
\text { Parameter }\end{array}$} & $\begin{array}{c}\text { Before } \\
\text { Treatment }\end{array}$ & $\begin{array}{c}\text { After } \\
\text { Treatment }\end{array}$ & $\begin{array}{c}\text { P- } \\
\text { value }\end{array}$ \\
\hline FSH $(\mathrm{mIU} / \mathrm{ml})$ & $3.87 \pm 1.8$ & $4.85 \pm 2.67$ & $>0.05$ \\
\hline LH $(\mathrm{mIU} / \mathrm{ml})$ & $9.17 \pm 2.84$ & $6.18 \pm 3.6$ & $<0.05^{*}$ \\
\hline $\begin{array}{l}\text { Testosterone } \\
(\mathrm{pg} / \mathrm{ml})\end{array}$ & $1.58 \pm 0.83$ & $1.38 \pm 0.44$ & $>0.05$ \\
\hline $\begin{array}{l}\text { Fasting insulin } \\
(\mu \mathrm{IU} / \mathrm{L})\end{array}$ & $14.3 \pm 4.3$ & $8.2 \pm 5.9$ & $<0.05^{*}$ \\
\hline
\end{tabular}

$\mathrm{P}$ value $<0.05$ significance

Table 4: Comparison between studied cases before and after metformin administration as regard LH/FSH ratio

\begin{tabular}{|l|c|c|c|c|}
\hline $\begin{array}{c}\text { Groups } \\
\text { Parameter }\end{array}$ & $\begin{array}{c}\text { Before } \\
\text { Treatment }\end{array}$ & $\begin{array}{c}\text { After } \\
\text { Treatment }\end{array}$ & $\begin{array}{c}\text { T } \\
\text { test }\end{array}$ & $\begin{array}{c}\text { P- } \\
\text { value }\end{array}$ \\
\hline $\begin{array}{l}\text { LH/FSH } \\
\text { ratio }\end{array}$ & $1.95 \pm 0.14$ & $0.38 \pm 0.07$ & 7.6 & $>0.05^{*}$ \\
\hline
\end{tabular}

Pathological findings: Assessment of the endometrial specimens before metformin treatment in patients with PCOS was done. Histopathologic examination revealed features of early proliferative endometrium in 64 cases (Fig. 1), late proliferative in 20 cases, while the remaining 16 cases showed features of simple endometrial hyperplasia (Figs. 2,3). Evaluation of the histologic changes after metformin treatment was also done. Among the 64 cases with early proliferative endometrium, 60 cases showed histologic evidence of progression to a late proliferative endometrium, while the remaining 4 cases showed no detectable histomorphologic changes before or after metformin administration. Among the 20 cases diagnosed as a late proliferative endometrium before metformin treatment, 6 cases progressed to early secretory endometrium (Fig. 4), 6 cases progressed to mid-secretory phase and the remaining 8 cases progressed to a late secretory endometrium that indicated successful ovulation after metformin treatment. As regard to the 16 cases with a diagnosis of simple endometrial hyperplasia before therapy, regression of hyperplastic changes was observed in 6 cases after metformin therapy. Imunohistochemical results: As regard to IHC, we first compared endometrial AR expression in women with PCOS to that in normal fertile controls and the results revealed marked increase in AR expression in patients with PCO compared to the normal fertile controls $(p<0.004)$. The AR expression in each cell component of the endometrium (epithelial \& stromal cells) was compared in the proliferative and secretory phases and a significant increase was observed in both epithelial and stromal AR expression in the endometrium of women with PCOS compared to normal fertile controls. (AR expression in epithelial cells: $p<0.013$ for proliferative and $p<0.003$ for secretory phase), (AR expression in stromal cells: $\mathrm{p}<0.005$ for proliferative and $p<0.004$ for secretory phase). In the present study, the highest androgen receptor expression in patients with PCOS was observed in cases of simple endometrial hyperplasia (Fig. 5) (H-scores ranged from 210 to $260 \&$ the mean average was 245), followed by cases diagnosed as early proliferative endometrium (Fig. 6) (H-score ranged from 130 to 180 with mean average 155) and lastly cases of the late proliferative endometrium (Fig. 7) were ranged in $\mathrm{H}$-score from 90 to 110 (mean average was 98). As regard to AR expression after metformin treatment, moderate decrease in AR expression was noted in cases that progressed from early to late proliferative endometrium after treatment (60/64 cases) (Hscores ranged between 110-130 with mean average 120 after treatment as compared to H-score ranged from 130 to 180 with mean average 155 before 
treatment). The remaining 4 cases with no detectable histomorphologic changes showed no significant difference in AR expression before or after treatment (H-score ranged between 130 and 180 with mean average of 155 before treatment and $\mathrm{H}$-score range of 150-170 with mean average 160 after treatment). As regard to the cases that progressed from late proliferative endometrium to secretory endometrium, the overall staining was reduced in each cell type during secretory phase compared to proliferative phase. By using the semiquantitative H-SCORE assessment of immunohistochemical staining, both the stromal and epithelial components of the proliferative endometrium showing greater $\mathrm{AR}$ expression than endometrium in the secretory phase of treated cases $(\mathrm{P}=0.003)$. Among the 20 cases that progressed from the late proliferative to secretory endometrium after treatment, marked decrease in AR expression was noted in both early (6/20 cases) (H-score ranged from 10 to 20 with mean average 14 after treatment, compared to $\mathrm{H}$-score from 90 to 110 with mean average of 98 before metformin therapy) and mid-secretory cases (6/20 cases) (Hscore ranged between 7 and 12 with mean average 9 after treatment, in comparison to $\mathrm{H}$-score from 90 to 110 with mean average of 98 before metformin therapy), while AR immunonegativity was observed in the cases of late secretory endometrium (8/20) (H-score 0) (Fig 8). So, according to these results, the epithelial AR immunostaining was markedly decreased along with reduced stromal AR expression in the early to midsecretory phases, while in the late secretory phase, AR immunoreaction was depleted from endometrial epithelial and stromal cells. There was a significant negative correlation between AR immunostaining and endometrial thickness $(\mathrm{P}<0.002)$ in PCOS women with restored ovulation (20 cases). Regression of hyperplasia was noted in 6 out of the 16 cases diagnosed as simple endometrial hyperplasia and showed marked decrease in AR expression (H-score ranged between 80 and 100 with mean average 85 after treatment compared to $\mathrm{H}$-scores ranged from 210 to 260 with mean average of 245 before metformin therapy). The remaining 10 cases revealed only minimal to mild decrease in AR expression.

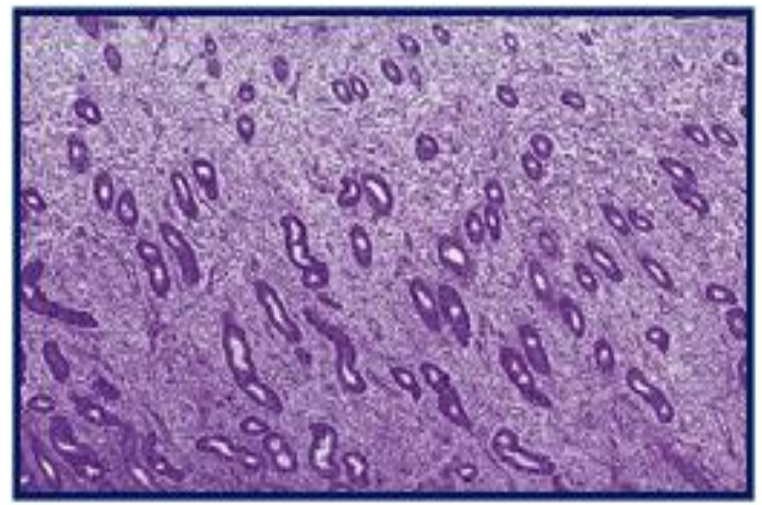

Figure 1: A case of early proliferative endometrium showing rounded uniform glands lined by proliferative epithelium (H\&E x100).

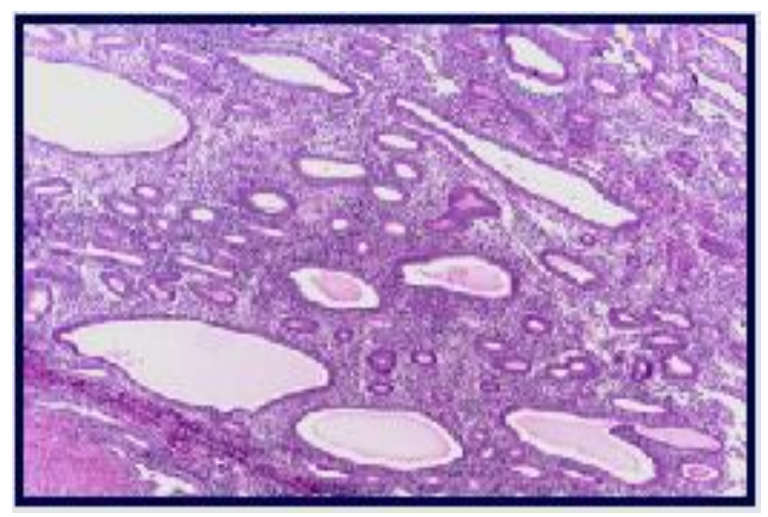

Figure 2: A case of simple endometrial hyperplasia showing crowded glands with occasional cystic dilatation (H\&E x 100).

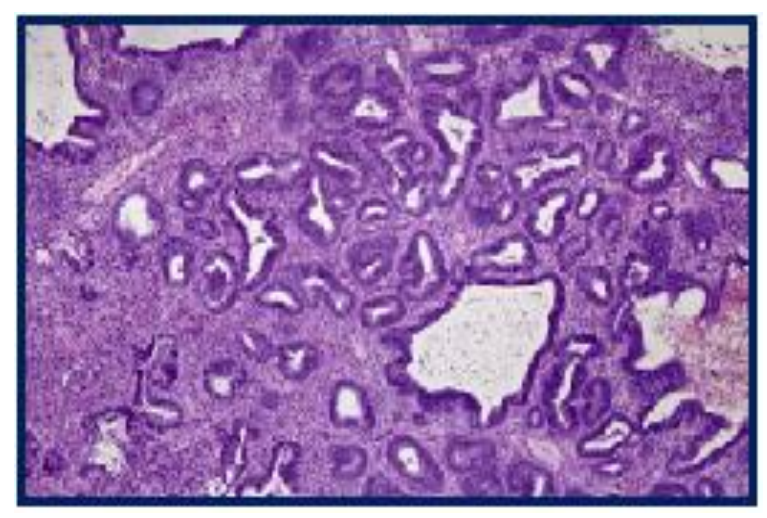

Figure 3: A case of simple endometrial hyperplasia showing crowded glands with occasional budding, branching and cystic dilatation (H\&E x100). 


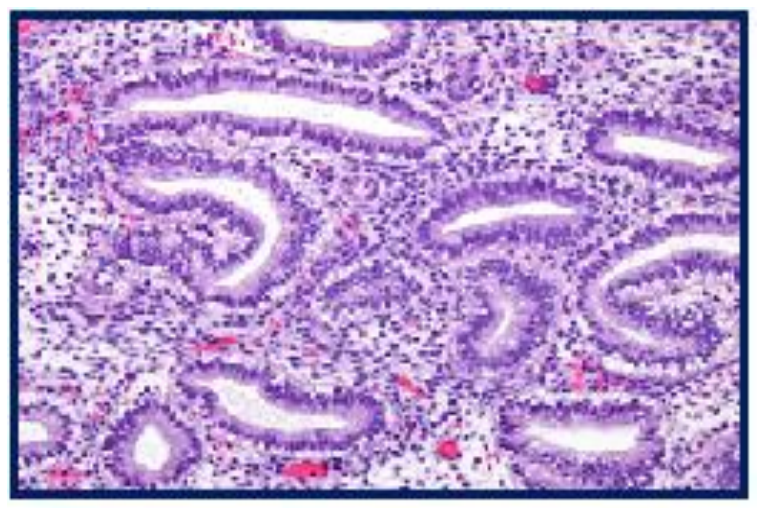

Figure 4: A case of early secretory endometrium showing crowded glands lined by single row of epithelium with sub nuclear vacuoles (H\&E x100).

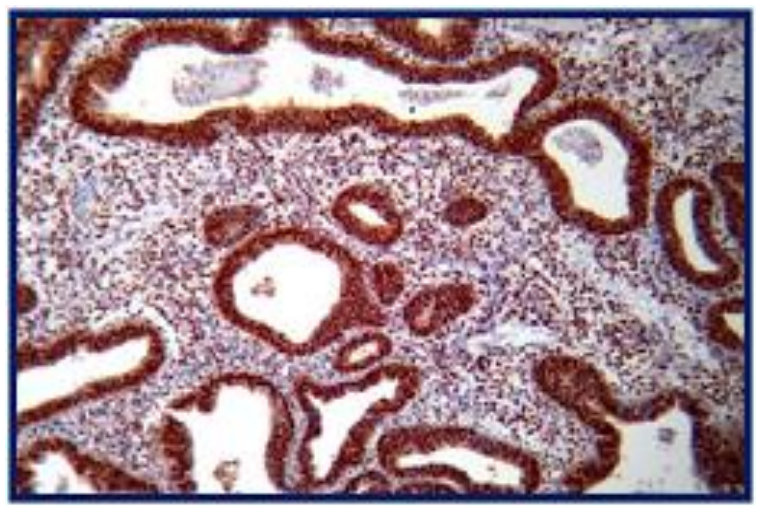

Figure 5: A case of simple endometrial hyperplasia showing strong and diffuse immunoreactivity to AR antibody in the glandular and stromal component. (DAB x 200 \& counterstained with Hx)

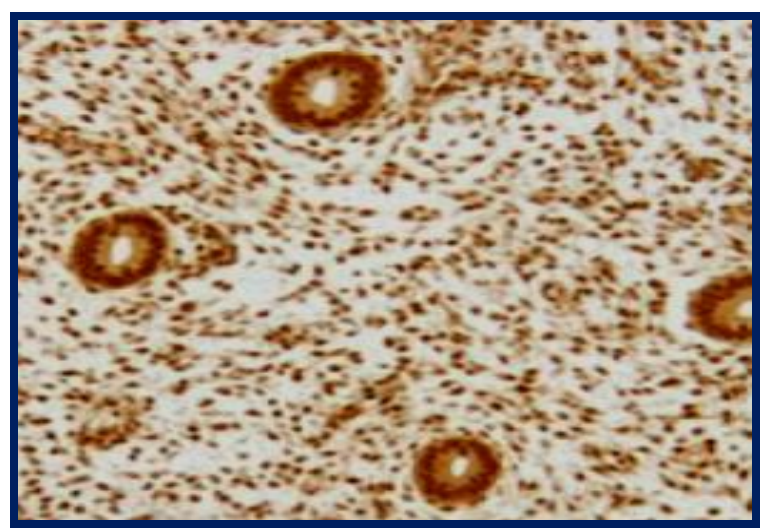

Figure 6: A case of early proliferative endometrium showing strong and diffuse immunoreactivity to AR antibody in the glandular and stromal component (DAB x 100 \& counterstained with Hx).

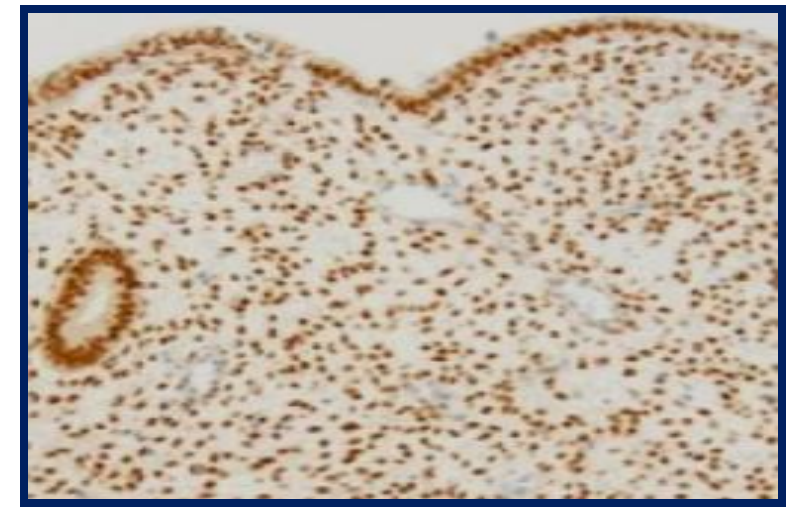

Figure 7: A case of late proliferative endometrium showing moderate and diffuse immunopositivity to AR antibody (DAB x 100 \& Counterstained with Hx).

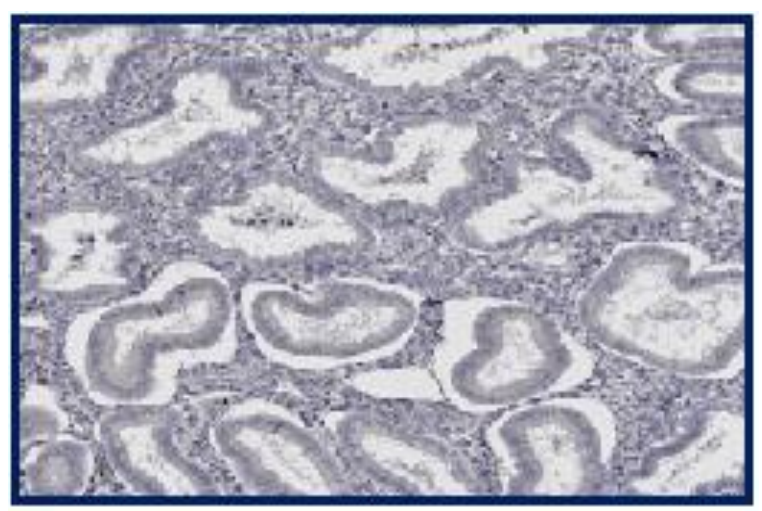

Figure 8: A case of late secretory endometrium showing immunonegativity for AR antibody (DAB x 200 \& counterstained with $\mathrm{Hx}$ ).

\section{DISCUSSION}

The accumulating evidences from previous clinical studies has shown that metformin administration improves menstrual cyclicity, increases ovulation and pregnancy rates, causes marked decrease in circulating insulin and androgen levels and also reduces insulin resistance in most women with PCOS ${ }^{(\mathbf{1 9})}$. The positive effects of metformin therapy appeared to be mediated by decreasing circulating insulin levels, increasing tissue-specific insulin sensitivity and reduction of ovarian androgen biosynthesis ${ }^{\left({ }^{19}\right)}$. Several clinical studies demonstrated that metformin can improve endometrial receptivity and enhance endometrial vascularity and blood flow in some women with PCOS ${ }^{(20)}$. The endocrine abnormalities associated with PCOS including dysregulation of gonadotropin-releasing hormone pulse generator to feedback inhibition by ovarian steroids that resulting in luteinizing hormone hypersecretion and decreased follicle-stimulating hormone and ovarian 
stromal-thecal hyperactivity which resulting in ovarian hyper-androgenism and all of which may lead to significant biochemical, reproductive and metabolic dysfunction in patients with PCOS ${ }^{\text {(21). }}$ In the present study, there was insignificant increase of FSH level, significant decrease of LH level, insignificant decrease of free testosterone level and significant decrease of fasting insulin level after metformin therapy, and these results showed agreement with those of Ganesan et al. ${ }^{(20)}$ who reported that metformin administration reduce fasting insulin level, testosterone and glucose levels in patients with PCOS. Metformin administration in patients with PCOS decreased serum fasting insulin, total and free testosterone as well as estradiol levels at oocyte retrieval, enhanced clinical pregnancy and live birth rates ${ }^{(20)}$. In the current study, the cases who had higher fasting insulin, had menstrual irregularity more than who had lower fasting insulin. A retrospective study performed by Gonzalez et al. ${ }^{(21)}$ showed that the higher degree of insulin resistance, the greater prevalence of amenorrhea in women having polycystic ovarian morphology. In contrast to our results, Mellembakken et al. ${ }^{(22)}$ found no difference in fasting insulin levels or insulin resistance between irregularly and regularly menstruating women with PCOS, although serum androgens were lower in the regularly menstruating group. In the current study, $20 \%$ of the investigated cases had regular menstrual cycle and ovulatory cycle after metformin therapy. Tosca $\boldsymbol{e t}$ al. (23) studied the effect of metformin administration in a dose of $500 \mathrm{mg} 3$ times/day for 8 weeks in 22 women with PCOS who suffered from chronic oligomenorrhea and found that 21 women reported regular menstruation at the close of the study, with $86 \%$ of these women having ovulatory progesterone levels. Kang and Paek ${ }^{(2)}$ reported that $26 \%$ of patients with PCOS treated with metformin had the cumulative ovulation rate $75 \%(6 / 8)$ at 24 months follow up. Metformin was considered better than placebo for ovulation rate in overall women with PCOS (OR 2.12 [1.50,3.00] $\mathrm{I} 2=69 \% \mathrm{p}=0.000019 ; 13$ studies, 875 participants), women with PCOS and a BMI $\leq 30 \mathrm{~kg} / \mathrm{m}^{2}$ (OR 2.33 [1.43-3.81] $\mathrm{I} 2=88 \% \quad \mathrm{p}=0.00071 ; 4$ studies, 417 participants), women with PCOS and a BMI $\geq 30 \mathrm{~kg} / \mathrm{m} 2 \quad(\mathrm{OR} \quad 1.94 \quad[1.20-3.15] \quad$ I $2=39 \%$ $\mathrm{p}=0.0073$; 9 studies, 458 participants) ${ }^{(23)}$ ) and women with non clomiphene citrate resistant
PCOS (OR 3.55 [1.46-8.65], 6 studies, 401 participants) ${ }^{(23)}$. However, there was significant statistical heterogeneity in overall women with PCOS and women with PCOS and a BMI $\leq 30 \mathrm{~kg} / \mathrm{m}^{2}$. There was no considerable difference in ovulation rate between metformin and placebo in women with clomiphene citrate resistant PCOS. In the current study, the mean BMI was $25.3 \pm 0.27$ $\mathrm{kg} / \mathrm{m}^{2}$ which decreased after metformin administration to $16.3 \pm 0.28 \mathrm{~kg} / \mathrm{m}^{2}$. Aurelian and Anca (13) reported that metformin therapy administered in high doses (3000 mg/day) on a 6 months period was useful for weight loss as BMI levels decreased significantly in the first 3 months in metformin group $\left(32.5+/-1.65 \mathrm{~kg} / \mathrm{m}^{2}\right.$ vs. $28.4+/-$ $1.95 \mathrm{~kg} / \mathrm{m}^{2}$ ), BMI variation in the non metformin group was smaller and BMI levels had reached a plateau after 3 months of metformin treatment. In contrast, Afroz et al. (25) found that in basal conditions, there was no difference between BMI in control group $(\mathrm{M} \pm \mathrm{SD} ; 27.14 \pm 3.89)$ and treated group (27.33 \pm 3.52$)$ and reported that during the 3 months pharmacological treatment with metformin, the mean BMI did not change in any group (control $26.97 \pm 3.91$ vs treated 27.13 $\pm 3.46, p=0.848$ ) although there was a slight reduction in BMI of both groups. In the present study, $10 \%$ of studied cases got pregnant after metformin adminestration. National Health and Medical Research Council (24) reported that metformin was considered better than placebo for pregnancy rate in most women with PCOS (OR $3.86[2.18,6.84] \mathrm{I} 2=0 \% \mathrm{p}<0.00001 ; 6$ studies, 479 participants) and women with PCOS and a BMI $\leq 30 \mathrm{~kg} / \mathrm{m}^{2}$ (OR 4.41 [2.24-8.66] $\mathrm{I} 2=40 \% \mathrm{p}=0.000017 ; 3$ studies, 250 participants) but no difference in women with PCOS and a BMI $\geq 30 \mathrm{~kg} / \mathrm{m}^{2}$, women with clomiphene citrate naïve PCOS, women with clomiphene citrate resistant PCOS or women with non clomiphene citrate resistant PCOS. Androgens are known to inhibit endometrial proliferation and differentiation via the nuclear androgen receptor (AR) ${ }^{(26)}$. In the current study, we found that women with PCOS exhibited marked increase in endometrial AR expression compared to normal fertile controls and this increase was apparent in glandular epithelium than stromal cells. Also, Cloke and Christian (6) reported that epithelial AR is up-regulated by estrogens and androgens but down-regulated by progesterone, and women with PCOS exhibit a higher mRNA and protein expression of AR in the 
epithelial cells of the endometrium compared to controls, and these results suggest that the poor reproductive performance observed in women with PCOS may be due to the concomitant increase in both serum androgens and elevations in endometrial AR. Also, Villavicencio et al. (26) found that the endometrial stromal and epithelial cells show a 30\% higher expression of estrogen receptor (ER) and similar expression of AR in women with PCOS compared to normal women. In the present study, we found higher expression of $\mathrm{AR}$ in the proliferative than the secretory phase and AR immunonegativity was observed in the late secretory phase. These results were in agreement with the results of Villavicencio et al. ${ }^{(26)}$ who reported more AR expression in the proliferative phases than in the secretory phases and in the late secretory phase there was no immunostaining in any of the cell types of the endometrium. Shang $\boldsymbol{e t}$ al. ${ }^{(7)}$ believed that androgens act as antagonists of estrogen and that high levels adversely affect priming of the endometrium in the follicular phase or development in the luteal phase. In addition to opposing the action of estrogen at its receptors, androgen may affect the endometrium directly through its own receptor, which has been shown to be expressed by the human endometrium ${ }^{(26)}$. In the current study and before metformin therapy, the endometrial protein expression of $\mathrm{AR}$ in the anovulatory women (64 cases), was markedly higher than in the controls, but in the PCOS women with restored ovulation and confirmed luteal phase after metformin therapy (20 cases), AR expression was in between those of the controls and the anovulatory PCOS women. Also in the current study, there was a significant negative correlation between AR immunostaining and endometrial thickness in the PCOS patients who restored ovulation after metformin administration. These data were in agreement with a previous report ${ }^{(25)}$. The prevalence of endometrial hyperplasia in women with PCOS varies greatly in the literature between $1 \%$ and $48.8 \%$, but risk of endometrial carcinoma was well found ${ }^{(27)}$, as women with PCOS possess a threefold increased risk of developing endometrial carcinoma when compared to the non-PCOS population ${ }^{(26)}$. However, the established treatment for endometrial hyperplasia (progestogens) involves multiple side effects and leaves the risk of recurrence. Previous studies ${ }^{(27)}$ demonstrated that metformin is considered the most commonly oral hypoglycaemic agent used in type II diabetes mellitus but has also been linked to the reversal of endometrial hyperplasia and may therefore contribute to decreasing the prevalence of endometrial carcinoma without the fertility and side effect consequences of current therapies (27), however, the efficacy and safety of metformin being used for this therapeutic target is unclear ${ }^{(28)}$. In the current study, regression of endometrial hyperplasia was noted in 6 out of the 16 cases after metformin therapy. Naomi et al. ${ }^{(28)}$ found that $62 \%$ of women with PCOS had regularization of their menses after metformin therapy, and therefore metformin may reduce the endometrial hyperplasia. The over expression of endometrial ARs in PCOS, as a result of estrogenic and androgenic stimulation, provides tangible evidence linking the abnormal hormonal milieu of PCOS with endometrial dysfunction ${ }^{(7)}$. Zhang and Liao ${ }^{(29)}$ demostrated that the high androgen levels could cause insulin resistance in endometrial glandular epithelial cells, and metformin has the effect of reversing this insulin resistance caused by androgens. Dumesic and Richards ${ }^{(30)}$ found that androgens inhibit the expression of ERs and PRs, but in PCOS it is believed that the stimulatory effects of estrogen on ER and PR expression outweigh androgenic inhibition.

\section{CONCLUSION}

Metformin therapy in patients with PCOS restores normal menstrual cyclicity, induces ovulation and showing decrease in LH level, fasting insulin and endometrial AR expression.

\section{CONFLICTS OF INTEREST}

There are no conflicts of interest.

\section{REFERENCES}

1. Broekmans F J, Knauff E A, Valkenburg O, Laven J S and Eijkemans M J (2006): PCOS according to the Rotterdam consensus criteria. Change in prevalence among WHO, anovulation and association with metabolic factors. An International Journal of Obstetrics and Gynaecology, 113: $1210-1217$. 
2. Kang J and Paek M (2017): Treatment of polycystic ovary syndrome with insulin sensitizer. Pediatric and Adolescent Medicine, 2 (4): 58-61.

3. Peigne $M$ and Dewailly $D$ (2014): Long term complications of polycystic ovary syndrome (PCOS). Ann. Endocrinol., 75(4): 194-199.

4. Allahbadia G N and Merchant R (2011): Polycystic ovary syndrome and impact on health. Middle East Fertility Society Journal, 16 (1): 19-37.

5. Apparao KB, Lovely LP, Gui Y, Lininger RA and Lessey BA (2002): Elevated endometrial androgen receptor expression in women with polycystic ovarian syndrome. Biol. Reprod., 66 (2):297-304.

6. Cloke B and Christian M (2012): The role of androgens and the androgen receptor in cycling endometrium. Mol. Cell. Endocrinol., (2):166-175.

7. Shang K, Jia X, Qiao J, Kang J and Guan $Y$ (2012): Endometrial abnormality in women with polycystic ovary syndrome. Reprod. Sci., 19 (7):674-683.

8. Huang A, Brennan $K$ and Azziz R (2010): Prevalence of hyperandrogenemia in the polycystic ovary syndrome diagnosed by the National Institutes of Health 1990 criteria. Fertil. Steril., 93: 1938-1941.

9. Ulku B, Mustafa B, Sibel K, Ayhan C and Sener G (2006): Use of an aromatase inhibitor in patients with polycystic ovary syndrome: a prospective randomized trial. Fertility and Sterility, 86 (5):1447-1451

10. Knight P G and Glister C (2003): Local roles of TGF-beta superfamily members in the control of ovarian follicle development. Anim. Reprod. Sci., 78: 165-183.

11. Dhindsa G, Bhatia $R$, Dhindsa $M$ and Bhatia V (2004): Insulin resistance, insulin sensitization and inflammation in polycystic ovarian syndrome. J. Postgrad. Med., 50:140-144.
12. Yashasvi S, Nivedita $M$, Pramila $K$ and Shivamurthy M (2016): Comparison of efficacy of metformin and oral contraceptive combination of ethinyl estradiol and drospirenone in polycystic ovary syndrome, Journal of the Turkish German Gyneocological Association, 17: 6-9.

13. Aurelian E R and Anca P C (2017): High dose metformin effect on weight loss, androgen levels and thyroid function in obese hypothyroid patients with PCOS. Endocrine Abstracts. 49 :1132-1153.

14. Glintborg D, Altinok M L, Mumm H, Hermann A P, Ravn $P$ and Andersen $M$ (2014): Body composition is improved during 12 months' treatment with metformin alone or combined with oral contraceptives compared with treatment with oral contraceptives in polycystic ovary syndrome. J. Clin. Endocrinol. Metab., 99 (7): 2584-2591.

15. Rui W and Ben Willem J (2017): The Rotterdam criteria for polycystic ovary syndrome: evidence-based criteria. Human Reproduction, 32 (2): 261-264.

16. Escobar H F, Carmina E, Dewailly D, Gambineri A, Kelestimur F, Moghetti P et al. (2012): Epidemiology, diagnosis and management of hirsutism: A consensus statement by the Androgen Excess and Polycystic Ovary Syndrome Society. Hum. Reprod. Update, 18:146-170.

17. Noyes $R$ N, Hertig A $\mathbf{T}$ and Rock $J$ (1950): Dating the endometrial biopsy. Fertil. Steril., 1: 3-25.

18. Hulchiy M, Nybacka A, Sahlin $L$ and Hirschberg AL (2016): Endometrial expression of estrogen receptors and the androgen receptor in women with polycystic ovary syndrome. J. Clin. Endocrinol., 101(2):561-71.

19. Nestler JE (2008): Metformin for the treatment of the polycystic ovary syndrome. N. Engl. J. Med., 358:47-54. 
20. Ganesan D, Palaniswamy S, Palanisamy $P$, Ponnusha $B$ S and Athimoolam A (2011): Effect of metformin combined therapy in patients with polycystic ovary syndrome. Int. J. Nutr. Pharmacol. Neurol. Dis., 1(2): 116-125.

21. Gonzalez CA, Nahum HP, Mendoza $R$ and Ayala AR (2003): Correlation between menstruation disorders and insulin resistance. Ginecol. Obstet. Mex., 71: 312317.

22. Mellembakken J R, Berga $S$ L, Kilen M, Tanbo T G, Abyholm T and Fedorcsák $P$ (2011): Sustained fertility from 22 to 41 years of age in women with polycystic ovarian syndrome. Hum. Reprod., 26 (9): 2499-2504.

23. Tosca L, Christelle R, Christine C, Sophie $T$ and Dupont $J$ (2010): Metformin decreases IGF1-induced cell proliferation and protein synthesis through AMPactivated protein kinase in cultured bovine granulosa cells. Society for Reproduction and Fertility, 139: 409-418.

24. Teede HJ, Misso ML, Costello MF, Dokras A, Laven J, Moran L, Piltonen T, Norman RJ (2018): Recommendations from the international evidence-based guideline for the assessment and management of polycystic ovary syndrome. Clin Endocrinol., doi: 10.1111/cen.13795.

25. Afroz M, Khanom A, Laila $R$ and Anwar B (2017): The effect of administration of metformin on BMI and insulin resistance in patients with polycystic ovary syndrome. J Gynecol Women's Health, 6(3): 1-5.

26. Villavicencio A, Bacallao K, Avellaira C, Gabler F, Fuentes A and Vega M (2006): Androgen and estrogen receptors and coregulators levels in endometria from patients with polycystic ovarian syndrome with and without endometrial hyperplasia. Gynecol. Oncol.,103(1):307-314.

27. Haoula Z, Salman $M$ and Atiomo W (2012): Evaluating the association between endometrial cancer and polycystic ovary syndrome. Hum. Reprod. Oxf. Engl., 27:1327-1331.
28. Naomi $S$, Thomas $R$ W Oliver, Hunain $S$, Juliane R F, Caroline A and William A (2016): Metformin for endometrial hyperplasia. doi: 10.1136/bmjopen-2016013385.

29. Zhang $L$ and Liao $Q$ (2010): Effects of testosterone and metformin on glucose metabolism in endometrium. Fertil. Steril., 93: 2295-2298.

30. Dumesic DA and Richards J S (2013): Ontogeny of the ovary in polycystic ovary syndrome. Fertil. Steril., 100 (1): 23-38. 